\title{
Partial and Total Lower Lid Reconstruction: Our Experience with 41 Cases
}

\author{
Paolo Fogagnolo ${ }^{a}$ Giacomo Colletti $^{b}$ Davide Valassina ${ }^{b}$ Fabiana Allevi $^{\mathrm{b}}$ \\ Luca Rossettic \\ ${ }^{a}$ G.B. Bietti Foundation for Study and Research in Ophthalmology - IRCCS, Rome, and ${ }^{b}$ Department of Maxillofacial \\ Surgery and 'Eye Clinic, San Paolo Hospital, University of Milan, Milan, Italy
}

\section{Key Words}

Lower lid reconstruction • Local flaps · Mucosal graft

\begin{abstract}
Purpose: To report our experience on lid reconstruction in patients with epitheliomas. Methods: A total of 41 consecutive patients affected by basal cell $(n=32)$ or squamous cell carcinoma $(n=9)$ underwent partial $(n=35)$ or total $(n=6)$ surgical demolition of the lower lid. Surgical defects $<25 \%$ $(n=10)$ received direct closure. If the defect involved 30 $60 \%$ of the eyelid $(n=21)$, a Tenzel semicircular flap or lateral advancement flap with a free mucosal graft was used. If the entire lid or a major part of the outer lamella had to be reconstructed $(n=10)$, a cheek advancement flap was used, with a free mucosal graft if the posterior lamella was involved. The success rates and the cosmetic and functional results were evaluated. Results: All 21 flaps used for partial reconstruction remained viable, whereas 1 of the 10 cheek flaps developed partial distal necrosis. Of the 27 mucosal grafts, 2 had to be removed for total necrosis, and 2 developed partial necrosis. In all cases, normal lid function and acceptable cosmetic results were obtained. Complications occurring in 4 cases (1 ectropion and 3 epiphora) were successfully managed with appropriate surgical procedures. Conclusions: Local flaps are the gold standard for lower lid
\end{abstract}

\section{KARGER}

Fax +4161306 1234

E-Mail karger@karger.ch

www.karger.com
(C) 2012 S. Karger AG, Basel

$0030-3755 / 12 / 2284-0239 \$ 38.00 / 0$

Accessible online at:

www.karger.com/oph reconstruction as they are highly reliable and guarantee optimal results. The technical details described in this study can help in achieving such results.

Copyright $\odot 2012$ S. Karger AG, Basel

\section{Introduction}

Eyelid tumors are frequent and their management is largely variable depending on size, location and histological patterns. Epitheliomas, the most common facial tumors, more frequently involve the lower than the upper lid. The availability of surrounding tissues is scarce for upper lid reconstructions; therefore, reconstructions of the upper lid are rarely reported in the literature and they may sometimes require technically demanding flaps $[1$, 2]. Conversely, a number of techniques have been described to reconstruct defects involving the lower lid [3-9].

As a general rule, if the involvement is $<25 \%$ of the entire lower lid or only the anterior lamella is affected, a simple skin graft or direct reapproximation is recommended $[1,10]$. When dealing with larger defects, strategies can largely vary and the most appropriate reconstruction technique has to be chosen among the numerous ones available in the literature $[1,3-11]$. 


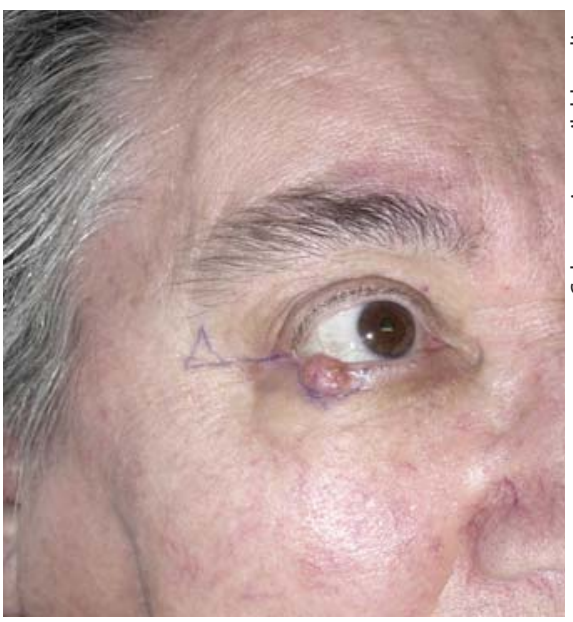

Fig. 1. Patient 1. Basal cell carcinoma affecting the lateral part of the lower lid. A preoperative drawing was made to plan the removal of the tumor and the reconstruction with a simple lateral advancement flap and a mucosal graft.

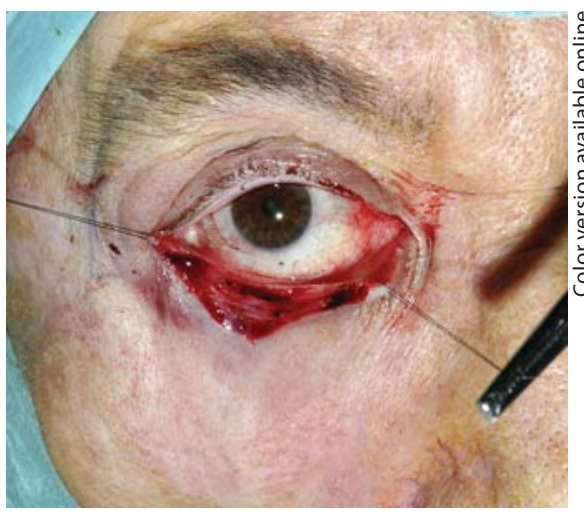

Fig. 2. Patient 1. Intraoperative detail: nearly $50 \%$ of the lid had to be removed.

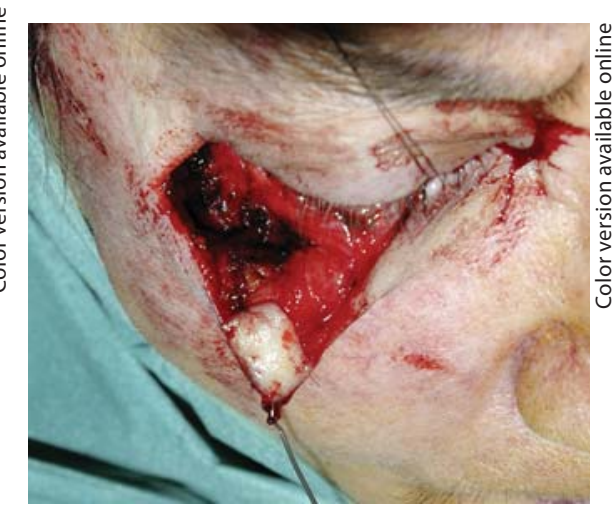

Fig. 3. Patient 1. Intraoperative detail: the inner lamella was reconstructed with a palatal mucosal graft.
The aim of this study is to present our data on the management of inferior eyelid tumors and to suggest a protocol for lower lid reconstruction which may help in obtaining more predictable results.

\section{Patients and Methods}

Between 2002 and 2011, a total of 41 Caucasian patients underwent lower eyelid surgery for skin epitheliomas. The cohort comprised 28 males and 13 females with an average age of 52 years (range 31-87). The lids were affected by basal cell carcinomas in 32 patients and by squamous cell carcinoma in 9 patients. After signing an informed consent, patients underwent surgery with local $(n=10)$ or general $(n=31)$ anesthesia.

The following surgical protocol was applied:

- Group 1: if the lesion involves $\leq 25 \%$ of the anterior lamella, skin plasty or skin graft is chosen.

- Group 2: if $<25 \%$ of the entire lid is lost, reconstruction with direct suture is performed.

- Group 3: if the defect involves 30-60\% of the eyelid, a Tenzel semicircular flap $[9,10]$ or simple advancement flap $[10,11]$ from the lateral remaining lid stump with canthotomy and cantholysis is chosen. A mucosal graft either from the oral mucosa or the nasal septal mucosa is performed to repair an eventual posterior defect.

- Group 4: if the lesion is $>60 \%$, a Mustardé flap $[1,3,10,12]$ is used; an oral mucosal graft is used to repair eventual posterior defects.

Functional and esthetic outcomes and complications were evaluated at 3 and 12 months.

\section{Results}

The distributions of our cases in the protocol groups $1,2,3$, and 4 were, respectively, 1, 9, 21, and 10. In group 3 (defects of 30-60\%), a Tenzel semicircular flap was used in 11 cases and a simple advancement flap with canthotomy and cantholysis in 10 cases; a mucosal graft was harvested from the oral mucosa in 18 cases, and from the nasal septal mucosa in 3 cases (fig. 1-6). In group 4 (fig. 7-11), a posterior graft was required in 6 cases.

All 11 semicircular flaps and the 10 lateral advancement flaps remained completely viable. Overall, in group 3 , we obtained a complete success rate of $100 \%$ in lower lid reconstruction. Of the 10 Mustardé flaps performed to reconstruct the entire lower lid, 1 flap developed partial necrosis of the distal part. In this case, we performed debridement of the nonviable tissue $(15 \times 8 \mathrm{~mm})$ and allowed the tissue to heal by secondary intention. In group 4 , we therefore obtained an overall success rate of $100 \%$ ( $90 \%$ complete success and $10 \%$ partial success). Of the 27 mucosal grafts, $2(7.4 \%)$ were lost totally and another 2 only partially. For the mucosal grafts, we had an overall success rate of $93.6 \%$ (85.2\% complete success and $7.4 \%$ partial success). None of the patients showed postoperative lagophthalmos or other postoperative complications.

Complications occurred in 4 cases, and 3 of the 9 patients who had their lower lid completely removed and reconstructed with a Mustardé flap showed epiphora at 3 


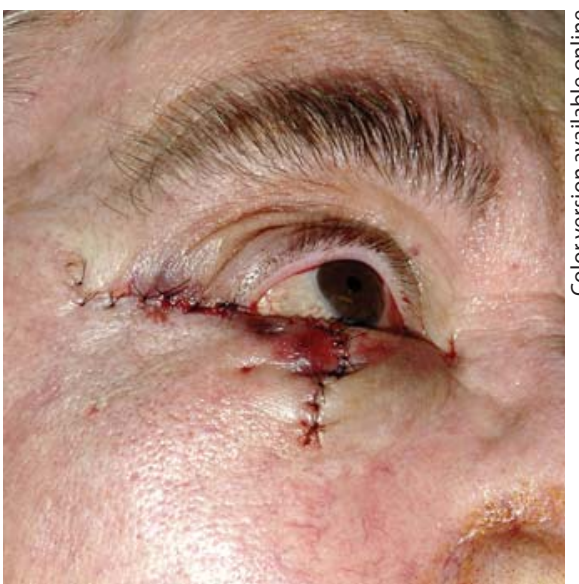

Fig. 4. Patient 1. The flap was advanced and sutured in a layered fashion.

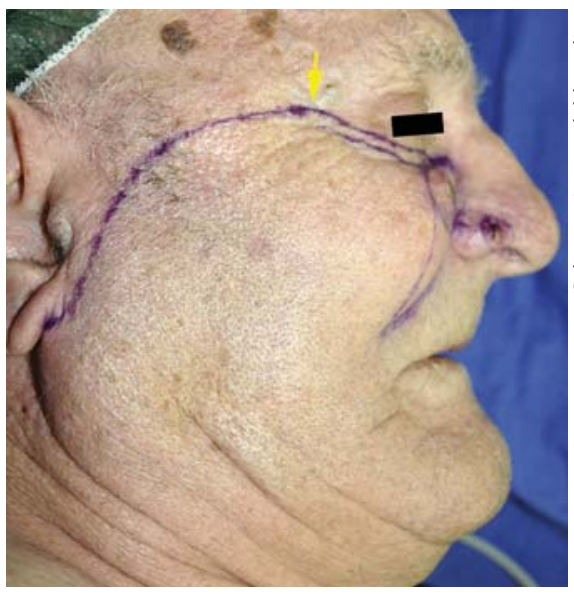

Fig. 7. Patient 2. Cheek flap for lower lid reconstruction. The horizontal limb of the flap (arrow) which will reconstruct the lid has to be superior to the lateral canthus to avoid lid retraction.

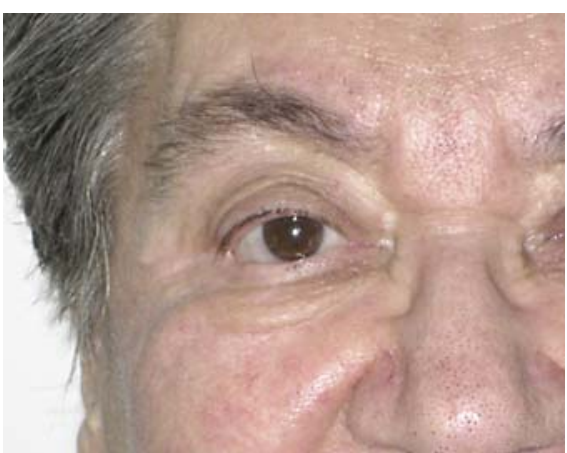

Fig. 5. Patient 1 . Postoperative result: an acceptable cosmetic result was obtained. No lagophthalmos is seen.

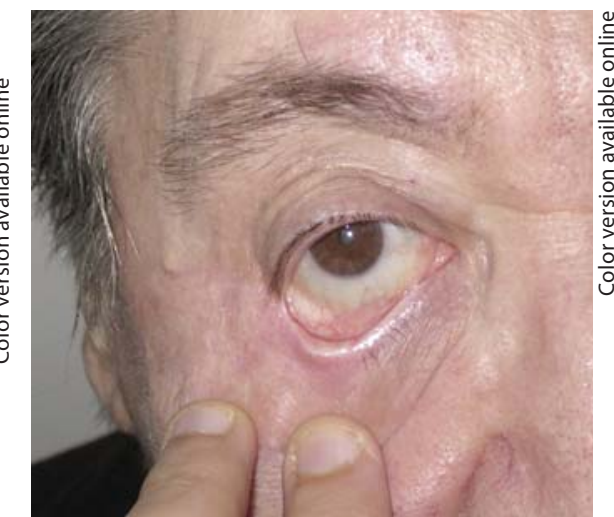

Fig. 6. Patient 1. Postoperative result: reconstruction of the inner lamella.

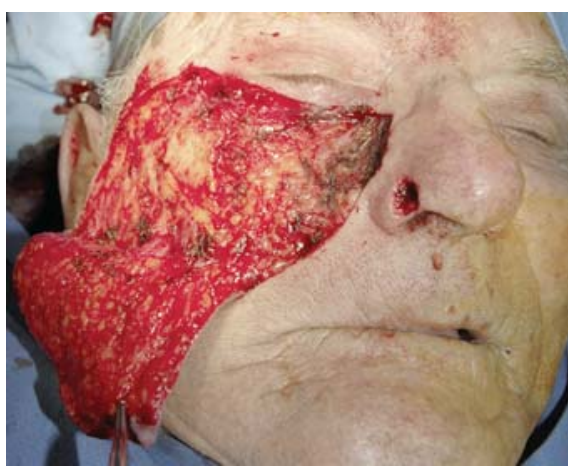

Fig. 8. Patient 2. Harvested cheek flap. Care has to be taken to sculpt the flap in the subcutaneous tissue to avoid damage to the facial nerve.

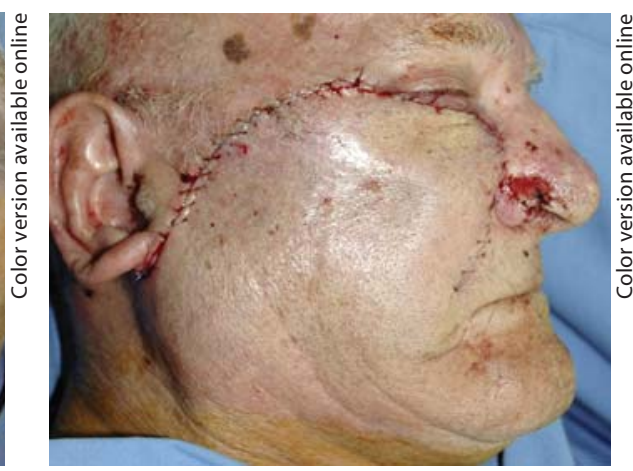

Fig. 9. Patient 2. Sutured flap. It has to be completely free from tension to avoid vascular injury. months postoperatively. This complication is due to the excision of the inferior lacrimal drainage system involved by malignancy. Epiphora was successfully treated with dacryocystorhinostomy.

The patient who was treated by resection and direct closure of the outer lamella developed lower lid ectropion and lagophthalmos. We successfully treated this secondarily with a double-pedicled upper lid flap (Tripier flap) [13]. Results were stable between 3 and 12 months.

\section{Discussion}

Surgical techniques for the excision of lower eyelid epitheliomas need to be critically evaluated and, if possible, standardized in order to achieve more predictable results.

In theory, when only the skin of the external lamella is involved, then simple grafts or direct closure can be sufficient. However, postoperative scar retraction should always be taken into account: if at the end of surgery any 


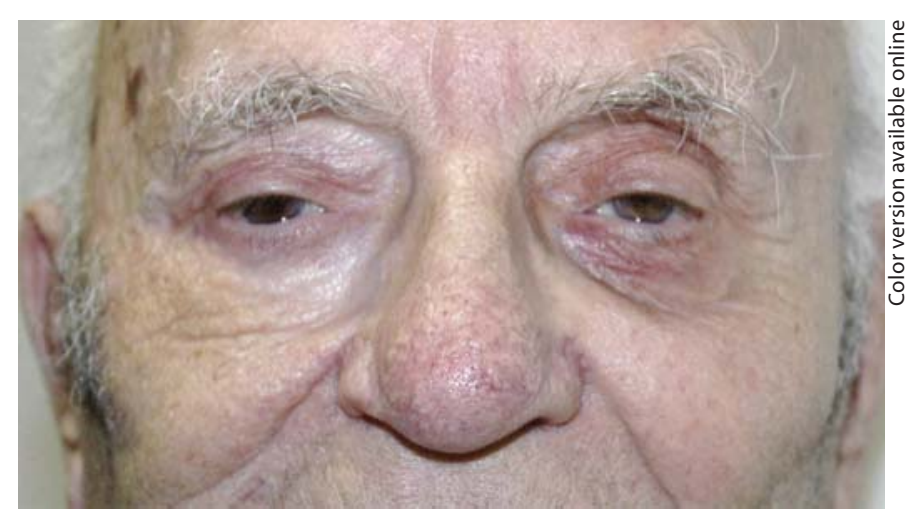

Fig. 10. Patient 2. Long-term postoperative result: an acceptable cosmetic result without lid retraction was obtained.

tension or distortion of the lower lid is evident, it is very likely that it will worsen over time, as we experienced in 1 patient. We treated this patient secondarily with a bipedicled upper lid flap. We preferred this to a skin graft because the former is less prone to contraction. Six months after this procedure, the ectropion and epiphora were completely resolved.

When a through-and-through defect results after tumor removal and the lid margin is part of the resection, other considerations need to be made. Firstly, the lower lid is a somewhat extensible structure; therefore, defects that involve up to $25 \%$ of the lid can be closed primarily as shown in the literature $[1,10]$. Secondly, if there is some tension during closure, a lateral cantholysis can be added to gain mobility [10]. Our experience with 9 cases confirms these findings.

When $30-60 \%$ of the lower lid is removed, a local flap reconstruction is needed. In the literature, a number of procedures for lower lid reconstructions are reported [1, 3-13]. We used a semicircular flap or simple lateral advancement flap in 31 cases, and we obtained total success in all cases. However, skin advancement does not address the internal lamella. Reconstruction of the conjunctival mucosa implies the use of another mucosa because everything else would cause corneal abnormalities. In general, we prefer the use of palatal mucosal grafts (fig. 3) due to their intrinsic stiffness that helps maintaining a lower lid position [4-6]. The nasal mucosa, though histologically more similar to the conjunctiva, is more delicate and does not contribute to lid suspension.

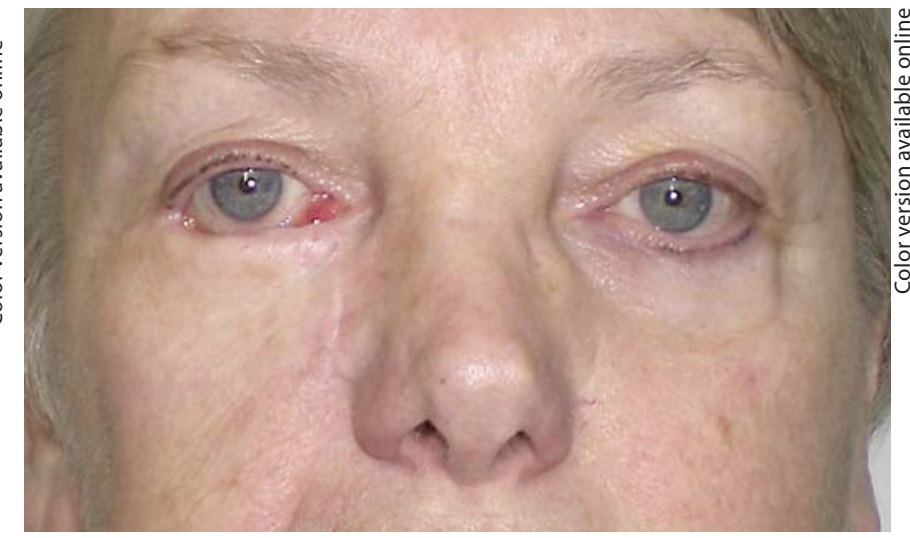

Fig. 11. Patient 3. Long-term postoperative result after complete removal of the lower lid and reconstruction with a cheek flap and palatal mucosal graft: an acceptable result with normal lid function was obtained.

The suspension of the newly created lower lid is of uttermost importance. Over time, all grafts have a variable degree of shrinkage. We routinely anchored the lateral part of the flap cranially to the periosteum of the lateral orbital rim with a 4-0 nonabsorbable nylon suture as a countermeasure to this phenomenon. By doing so, we did not observe any retraction of the reconstructed lid.

In our experience, the mucosal grafts still show a variable degree of survival. In our case series, we have observed an overall success rate of $93.6 \%$. In order to increase the chance of survival, we routinely suture the graft to the inner aspect of the skin flap meticulously. Also, we perform a lower lid cranial traction with 5-0 nylon sutures suspended to the forehead with dressing strips, and we leave them in place for 3-4 days. This procedure immobilizes the graft, thus increasing the chances of survival.

When the entire lower lid or a major part of the external lamella has to be reconstructed, the Mustardé cheek flap is the best option $[1,3,10,12]$, and our experience confirms this (overall success rate of $100 \%$ with only 1 case of partial failure of the distal aspect of the flap).

Few technical notes are worth mentioning for the cheek flap. Firstly, the arm of the flap running horizontally (which will reconstruct the lid) had to be drawn slightly cranially in order to have redundant tissues for the lid reconstruction and counterforce any secondary retraction (fig. 7).

Secondly, no tension has to be exerted on the flap, and thus one must be ready to extend the skin-releasing 
incision posterior to the auricle and even in the neck (fig. 9). However, it is not the increase in the width-tolength ratio that compromises the vascularity of this excellently reliable flap. Conversely, excessive tension would likely impair venous drainage and stimulate necrosis. As with smaller reconstructions, the suspension of the flap is of great importance. We routinely place a 4-0 nonabsorbable nylon suture from the lateral part of the flap to the periosteum of the lateral orbital rim. This helps in counteracting any retraction during the postoperative period.

\section{Conclusions}

Lower eyelid epitheliomas can be managed with a simple procedure that covers all needs for lower lid reconstruction. If attention is given to the single steps, then very good results are easily achievable.

\section{Disclosure Statement}

The authors have no conflicts of interest to disclose.

\section{References}

$\checkmark 1$ Rafii AA, Enepekides DJ: Upper and lower eyelid reconstruction: the year in review. Curr Opin Otolaryngol Head Neck Surg 2006;14:227-233.

-2 Brusati R, Colletti G, Redaelli V: Upper eyelid reconstruction with forehead galeal flap. J Plast Reconstr Aesthet Surg 2009;62:901905.

$\checkmark 3$ Callahan MA, Callahan A: Mustardé flap lower lid reconstruction after malignancy. Ophthalmology 1980;87:279-286.

4 Perry MJ, Langtry J, Martin IC: Lower eyelid reconstruction using pedicled skin flap and palatal mucoperiosteum. Dermatol Surg 1997;23:395-397; discussion 397-398.
5 Ito O, Suzuki S, Park S, Kawazoe T, Sato M, Saso Y, Iwasaki Y, Hata Y: Eyelid reconstruction using a hard palate mucoperiosteal graft combined with a V-Y subcutaneously pedicled flap. Br J Plast Surg 2001;54:106-111.

6 Miyamoto J, Nakajima T, Nagasao T, Konno E, Okabe K, Tanaka T, Fujii S, Kobayashi H: Full-thickness reconstruction of the eyelid with rotation flap based on orbicularis oculi muscle and palatal mucosal graft: long-term results in 12 cases. J Plast Reconstr Aesthet Surg 2009;62:1389-1394.

7 Wessels WL, Graewe FR, van Deventer PV: Reconstruction of the lower eye lid with a rotation-advancement tarsoconjunctival cheek flap. J Craniofac Surg 2010;21:17861789.

${ }_{8}$ Saito A, Saito N, Furukawa H, Hayashi T, Oyama A, Funayama E, Minakawa H, Yamamoto Y: Reconstruction of periorbital defects following malignant tumour excision: a report of 50 cases. J Plast Reconstr Aesthet Surg 2012;65:665-670
Tenzel RR, Stewart WB: Eyelid reconstruction by the semicircle flap technique. Ophthalmology 1978;85:1164-1169.

10 Codner MA, McCord CD, Mejia JD, Lalonde D: Upper and lower eyelid reconstruction. Plast Reconstr Surg 2010;126:231e-245e.

11 McGregor IA: Eyelid reconstruction following subtotal resection of upper or lower lid. Br J Plast Surg 1973;26:346-354.

12 Mustardé JC: The use of flaps in the orbital region. Plast Reconstr Surg 1970;45:146-150.

13 Tripier L: Lambeau musculo-cutané en forme de pont appliqué à la restauration des paupières. Gazette des Hôpitaux de Paris 1889;62:1124. 